PROCEEDINGS OF THE

AMERICAN MATHEMATICAL SOCIETY

Volume 129, Number 12, Pages 3447-3452

S 0002-9939(01)06293-1

Article electronically published on July 2, 2001

\title{
OBSTRUCTIONS TO DEFORMATIONS OF D.G. MODULES
}

\author{
TRINA ARMSTRONG AND RON UMBLE
}

(Communicated by Eric Friedlander)

\begin{abstract}
Let $\mathbf{k}$ be a field and $n \geq 1$. There exist a differential graded $\mathbf{k}$ module $(V, d)$ and various approximations to a differential $d+t d_{1}+t^{2} d_{2}+$ $\cdots+t^{n} d_{n}$ on $V[[t]]$, one of which gives a non-trivial deformation, another is obstructed, and another is unobstructed at order $n$. The analogous problem in the category of $\mathbf{k}$-algebras in characteristic zero remains a long-standing open question.
\end{abstract}

\section{INTRODUCTION}

Most deformation theories, including those of Froelicher-Nijenhuis-KodairaSpencer for complex analytic manifolds and of Gerstenhaber for algebras, introduce both a primary obstruction to extending an infinitesimal deformation as well as ones of successively higher order, which appear after each previous obstruction is passed. Somewhat surprisingly, examples of infinitesimals with a non-vanishing higher order obstruction are often difficult to find. This difficulty seems to increase with richer algebraic structure. In the deformation theory of Hopf algebras - a setting with rich algebraic structure -S. D. Schack's "primary obstruction conjecture" asserts that an infinitesimal extends to a deformation whenever its primary obstruction vanishes. In the more relaxed setting of finite dimensional associative algebras, Gerstenhaber and Schack [4] cleverly apply topological methods to produce infinitesimals with vanishing primary obstructions on certain rigid algebras in characteristic $p>0$. But these examples are by no means simple, and the analogous problem in characteristic zero remains open ([3], p. 61).

In this paper we consider the deformation theory of differential graded $\mathbf{k}$-modules (d.g.m.'s) over an arbitrary field k. In this setting, the algebraic structure is quite simple and infinitesimal deformations with non-vanishing higher order obstructions are easily observed. Given an integer $n \geq 1$, we exhibit a d.g.m. $(V, d)$ and various approximations to a differential $d+t d_{1}+t^{2} d_{2}+\cdots+t^{n} d_{n}$ on $V[[t]]$, one of which gives a non-trivial deformation, another is obstructed, and another is unobstructed at order $n$.

Classically, one finds the deformation theory of d.g.m.'s imbedded in richer theories such as the deformation theory of differential graded $\mathbf{k}$-algebras; as such, it has been considered by many authors (see for example [1], [2, 6], 7], 8]). Recently, Gerstenhaber and Wilkerson [5] gave a systematic treatment of the theory, whose

Received by the editors February 9, 1995.

2000 Mathematics Subject Classification. Primary 13D10.

This paper reports the results of an undergraduate honors project directed by the second author. 
relative simplicity provides an ideal entry point for the uninitiated reader. Many of the ideas and techniques familiar in more general settings appear here, but without the tight constraints that typically confound even the most basic computational examples. We begin with a review of the ideas we need.

\section{Cohomology And Deformations of D.G. Modules}

Let $R$ be a commutative ring with identity - in this discussion $R$ will be either a field $\mathbf{k}$ or the ring of formal power series $\mathbf{k}[[t]]=\left\{\sum \lambda_{i} t^{i} \mid \lambda_{i} \in \mathbf{k}\right\}$. Let $\left\{M_{p}\right\}_{p \in \mathbf{Z}}$ be a sequence of $R$-modules. Then $M=\sum_{p \in \mathbf{Z}} M_{p}$ is a graded $R$-module; an element $x_{p} \in M_{p}$ is said to be homogeneous of degree $p$, in which case we write $\left|x_{p}\right|=p$. Let $M$ and $N$ be graded $R$-modules. An $R$-linear map $f: M \rightarrow N$ has degree $p$ if $|f(x)|=|x|+p$ for each homogeneous $x \in M$, in which case we write $|f|=p$. The set of all such maps of degree $p$ is denoted by $\operatorname{Hom}_{R}^{p}(M, N)$. An $R$-linear map $d: M \rightarrow M$ of degree \pm 1 such that $d^{2}=0$ is a differential on $M$ and the pair $(M, d)$ is a differential graded $R$-module (d.g.m.).

Let $\left(V, d_{V}\right)$ and $\left(M, d_{M}\right)$ be a d.g.m. For each $p \in \mathbf{Z}$, define the $R$-module of $p$-cochains on $V$ with coefficients in $M$ by $C^{p}(V ; M)=H_{o m}^{-p}(V, M)$. Let $C^{*}(V ; M)=\sum_{p \in \mathbf{Z}} C^{p}(V ; M)$ and define the $p^{t h}$ coboundary map $\delta^{p}: C^{p}(V ; M) \rightarrow$ $C^{p+1}(V ; M)$ by

$$
\delta^{p}(f)=d_{M} f-(-1)^{p} f d_{V}
$$

Then

$$
\delta=\sum_{p \in \mathbf{Z}} \delta^{p} \in \operatorname{Hom}_{\mathbf{k}}^{1}\left(C^{*}(V ; M), C^{*}(V ; M)\right)
$$

and it is trivial to check that $\delta^{2}=0$ so that $\left\{C^{*}(V ; M), \delta\right\}$ is a d.g.m. The cohomology of $V$ with coefficients in $M$ is the cohomology of $\left\{C^{*}(V ; M), \delta\right\}$, i.e., the graded $R$-module $H^{*}(V ; M)=\sum_{p \in \mathbf{Z}} H^{p}(V ; M)$, where $H^{p}(V ; M)=$ $\operatorname{ker}\left(\delta^{p}\right) / \operatorname{Im}\left(\delta^{p-1}\right)$. The elements of $\operatorname{ker}\left(\delta^{p}\right)$ are the $p$-cocycles; the elements of $\operatorname{Im}\left(\delta^{p-1}\right)$ are the $p$-coboundaries. Two $p$-cocycles $f$ and $g$ are cohomologous provided $f-g \in \operatorname{Im}\left(\delta^{p-1}\right)$; the class $[f] \in H^{p}(V ; M)$ is called the cohomology class of $f$. When $V=M$ we let $C^{*}(V)=C^{*}(V ; V)$ and $H^{*}(V)=H^{*}(V ; V)$.

Let $(V, d)$ be a d.g.m. over a field k. The cohomology $H^{*}(V)$ directs the deformation theory of $(V, d)$ in the following way: Let $t$ be an indeterminant of degree 0 and imbed $V$ as the set of constants in the $\mathbf{k}[[t]]$-module of formal power series $V[[t]]=\left\{\sum t^{i} v_{i} \mid v_{i} \in V\right\}$. Extend $d$ to $V[[t]]$ via $d\left(\sum t^{i} v_{i}\right)=\sum t^{i} d\left(v_{i}\right)-$ this is the unique $\mathbf{k}[[t]]$-linear extension of $d$-and obtain the $d . g . \mathbf{k}[[t]]$-module $V_{0}[[t]]=(V[[t]], d)$. Given $\left\{d_{i} \in C^{1}(V)\right\}_{i \geq 1}$, extend each $d_{i}$ to $V[[t]]$ in the same way we extended $d$ and define

$$
d_{t}=d+t d_{1}+t^{2} d_{2}+t^{3} d_{3}+\cdots
$$

If $d_{t}$ is a differential on $V[[t]]$, then $V_{t}=\left(V[[t]], d_{t}\right)$ is a deformation of $(V, d)$ as a d.g. $\mathbf{k}[[t]]$-module. The trivial deformation $V_{t}=V_{0}[[t]]$ satisfies $d_{t}=d$, i.e., $d_{i}=0$ for all $i$. A deformation $V_{t}$ with differential $d_{t}$ such that $d_{n} \neq 0$ and $d_{i}=0$ for all $i>n$ is called a polynomial deformation of order $n$. 
Let $V_{t}=\left(V[[t]], d_{t}\right)$ be a deformation. Define $\mathcal{O}_{0}=0$ and for each $n \geq 1$ consider the 2-cochain

$$
\mathcal{O}_{n}=-\sum_{i=1}^{n} d_{i} d_{n-i+1}
$$

Expand the right-hand side of $d_{t} \circ d_{t}=0$ and equate coefficients to obtain the relations

$$
\left\{\delta\left(d_{n+1}\right)=\mathcal{O}_{n}\right\}_{n \geq 0}
$$

Observe that $d_{1}$ is a 1-cocycle and that $\mathcal{O}_{n}$ is a cobounding 2-cocycle for each $n$; these are necessary and sufficient conditions for an arbitrary series $d_{t}^{\prime}=d+$ $t d_{1}^{\prime}+t^{2} d_{2}^{\prime}+\cdots$ to be a differential, i.e., for $\left(V[[t]], d_{t}^{\prime}\right)$ to be a deformation. The differential $d_{t}$ is commonly referred to as a deformation of $d$; the 1-cocycle $d_{1}$ is commonly referred to as an infinitesimal deformation. The 2-cocycles $\left\{\mathcal{O}_{n}\right\}_{n \geq 1}$ are the obstructions to extending the linear approximation $d+t d_{1}$, and $\mathcal{O}_{1}$ is called the primary obstruction.

This suggests the following inductive strategy for constructing deformations: Given a d.g.m. $(V, d)$, choose a 1 -cocycle $d_{1}$ as required by the $0^{\text {th }}$ relation in (1) and obtain the linear approximation $d+t d_{1}$. Inductively, assume that for each $k \leq n$, some 1-cochain $d_{k}$ has been chosen so that $\delta\left(d_{k}\right)=\mathcal{O}_{k-1}$. If $\mathcal{O}_{n}$ fails to cobound, the approximation $d+t d_{1}+\cdots+t^{n} d_{n}$ is obstructed at order $n$ and the process terminates. Otherwise, choose a 1-cochain $d_{n+1}$ such that $\delta\left(d_{n+1}\right)=\mathcal{O}_{n}$ and extend the approximation to $d+t d_{1}+\cdots+t^{n+1} d_{n+1}$. If the process can be continued indefinitely, there is a differential $d_{t}=d+t d_{1}+t^{2} d_{2}+\cdots$ whose partial sums are the approximations obtained inductively. Indeed the inductive process can be continued indefinitely if $\mathcal{O}_{n} \in[0]$ in $H^{2}(V)$ for all $n$. Since this happens automatically whenever $H^{2}(V)=0$ we obtain:

Theorem 2.1. Let $(V, d)$ be a d.g.m. and let $d_{1}$ be a 1-cocycle. If $H^{2}(V)=0$, there exists a sequence of 1 -cochains $\left\{d_{i}\right\}_{i \geq 2}$ such that $\left(V[[t]], d_{t}=d+t d_{1}+t^{2} d_{2}+\cdots\right)$ is a deformation.

Two deformations $V_{t}=\left(V[[t]], d_{t}\right)$ and $V_{t}^{\prime}=\left(V[[t]], d_{t}^{\prime}\right)$ are equivalent if there exists a $\mathbf{k}[[t]]$-linear automorphism $\phi_{t}: V[[t]] \rightarrow V[[t]]$ such that

(1) $\phi_{0}=I d_{V[t]]}$ and

(2) $d_{t} \phi_{t}=\phi_{t} d_{t}^{\prime}$.

Condition (1) implies the existence of maps $\left\{\phi_{i} \in C^{0}(V)\right\}_{i \geq 1}$ such that $\phi_{t}=I d+$ $t \phi_{1}+t^{2} \phi_{2}+\cdots$. Condition (2), the naturality condition, implies that $\phi_{t}$ induces a $\mathbf{k}[[t]]$-linear isomorphism of d.g.m.s $H^{*}\left(V[[t]], d_{t}^{\prime}\right) \approx H^{*}\left(V[[t]], d_{t}\right)$. When $\phi_{t}$ exists we call it an equivalence and write $\phi_{t}: V_{t} \sim V_{t}^{\prime}$.

Suppose that $V_{t}$ and $V_{t}^{\prime}$ are deformations of $(V, d)$ and assume that $\phi_{t}: V_{t} \sim V_{t}^{\prime}$ is an equivalence. Expand and collect first order coefficients in relation (2) and obtain

$$
d_{1}^{\prime}-d_{1}=d \phi_{1}-\phi_{1} d=\delta\left(\phi_{1}\right) ;
$$

thus $d_{1}$ and $d_{1}^{\prime}$ are cohomologous. In particular, if $V_{t}^{\prime}$ is the trivial deformation $V_{0}[[t]]$, then $d_{1}^{\prime}=0$ in which case

$$
\delta\left(\phi_{1}\right)=-d_{1}
$$


A d.g.m. $(V, d)$ is rigid if every deformation $V_{t}$ is equivalent to the trivial deformation $V_{0}[[t]]$. We conclude this section with a standard theorem whose proof is included for completeness.

Theorem 2.2. Let $(V, d)$ be d.g.m. If $H^{1}(V)=0$, then $(V, d)$ is rigid.

Proof. Let $V_{t}=\left(V[[t]], d_{t}=d+t d_{1}+t^{2} d_{2}+\cdots\right)$ be a deformation; by assumption, there exists a 0 -cochain $\phi_{1}$ such that $\delta\left(\phi_{1}\right)=-d_{1}$. Consider the $\mathbf{k}[[t]]$-linear isomorphism $\phi_{t}^{(1)}=I d-t \phi_{1}$ and define $d_{t}^{(1)}=\phi_{t}^{(1)} d_{t}\left[\phi_{t}^{(1)}\right]^{-1}$. Then $\phi_{t}^{(1)}:\left(V[[t]], d_{t}^{(1)}\right) \sim V_{t}$ is an equivalence. Expanding $d_{t}^{(1)} \phi_{t}^{(1)}=\phi_{t}^{(1)} d_{t}$ and equating first order coefficients gives $d_{1}^{(1)}=\left(d \phi_{1}-\phi_{1} d\right)+d_{1}=0$, so the linear term in $d_{t}^{(1)}$ vanishes. Inductively, suppose that $V_{t}^{(r)}=\left(V[[t]], d_{t}^{(r)}\right)$ is a deformation with $d_{i}^{(r)}=0$ for all $i \leq r$ and that $\phi_{t}^{(r)}=\prod_{i=1}^{r}\left(I d-t^{i} \phi_{i}\right): V_{t}^{(r)} \sim V_{t}$ is an equivalence. Then $\delta\left(d_{r+1}^{(r)}\right)=0$, so by assumption there exists a 0-cochain $\phi_{r+1}$ such that $\delta\left(\phi_{r+1}\right)=$ $-d_{r+1}^{(r)}$. Define $\phi_{t}^{(r+1)}=\prod_{i=1}^{r+1}\left(I d-t^{i} \phi_{i}\right)$ and $d_{t}^{(r+1)}=\phi_{t}^{(r+1)} d_{t}^{(r)}\left[\phi_{t}^{(r+1)}\right]^{-1}$; then $d_{r+1}^{(r+1)}=\left(d \phi_{r+1}-\phi_{r+1} d\right)+d_{r+1}^{(r)}=0$ so that $d_{i}^{(r+1)}=0$ for all $i \leq r+1$ and $\phi_{t}^{(r+1)}:\left(V[[t]], d_{t}^{(r+1)}\right) \sim V_{t}$ is an equivalence. Hence there is a sequence of equivalences $\left\{\phi_{t}^{(r)}=\prod_{i=1}^{r}\left(I d-t^{i} \phi_{i}\right)\right\}_{r \geq 1}$ that $t$-adically converge to an equivalence $\phi_{t}^{(\infty)}=\prod_{r \geq 1}\left(I d-t^{r} \phi_{r}\right): V_{0}[[t]] \sim V_{t}$.

Analogs of Theorem 2.1 and Theorem 2.2 appear in the deformation theory of differential graded algebras with an apparent shift of dimension. This dimension shift, which reflects nothing more than a change in point of view, is discussed in [8]. We are ready for the construction promised.

\section{Examples of DEFormations AND OBSTRUCTED APPROXimations}

Let $\mathbf{k}$ be any field, let $V=\sum_{p \in \mathbf{Z}} V_{p}$ be a graded $\mathbf{k}$-module and choose a basis $\left\{x_{\alpha}\right\}$ for $V$. If $\left\{x_{\beta}\right\}$ is a set of vectors in $V$, let $\left\langle x_{\beta}\right\rangle$ denote the $\mathbf{k}$-linear span. Consider the associated graded k-module $\operatorname{Hom}_{\mathbf{k}}^{*}(V, V)=\left\langle x_{i} \frac{\partial}{\partial x_{j}}\right\rangle$, where

$$
x_{i} \frac{\partial x_{k}}{\partial x_{j}}=\left\{\begin{array}{cl}
x_{i}, & \text { if } j=k, \\
0, & \text { otherwise. }
\end{array}\right.
$$

Thus

$$
x_{i} \frac{\partial}{\partial x_{j}}\left(x_{k} \frac{\partial}{\partial x_{\ell}}\right)=x_{i} \frac{\partial x_{k}}{\partial x_{j}} \frac{\partial}{\partial x_{\ell}} .
$$

In particular, let $V_{p}=\{0\}$ for all $p \leq 0$ and $V_{p}=\left\langle x_{2 p-1}, x_{2 p}\right\rangle$ for all $p \geq 1$; define

$$
d=\sum_{i=1}^{\infty} x_{6 i-5} \frac{\partial}{\partial x_{6 i-3}} .
$$

Clearly $d^{2}=0$ so that $(V, d)$ is a d.g.m. Fix a positive integer $n \geq 2$ and define

$$
d_{1}=x_{1} \frac{\partial}{\partial x_{4}}+\sum_{i=1}^{n-1} x_{6 i-2} \frac{\partial}{\partial x_{6 i}} .
$$


Since $\delta\left(d_{1}\right)=0$, the expression $d+t d_{1}$ is a linear approximation to a deformation. Furthermore,

$$
\delta\left(-x_{3} \frac{\partial}{\partial x_{6}}\right)=-x_{1} \frac{\partial}{\partial x_{6}}=-d_{1}^{2}=\mathcal{O}_{1},
$$

so the primary obstruction vanishes in cohomology. For $2 \leq k \leq n$ define

$$
d_{k}=-x_{6 k-9} \frac{\partial}{\partial x_{6 k-6}}+\left(1-\delta_{n, k}\right) x_{6 k-5} \frac{\partial}{\partial x_{6 k-2}},
$$

where $\delta_{n, k}$ is the Kronecker delta, and consider the subspaces $S=\left\langle x_{2 i}\right\rangle_{i \geq 1}$ and $S^{\perp}=\left\langle x_{2 i-1}\right\rangle_{i>1}$ spanned by basis elements of even and odd index, respectively. Note that $d_{i}$ is supported on $S$ for $1 \leq i \leq n$; and furthermore, $d_{i}$ takes values in $S^{\perp}$ for $2 \leq i \leq n$; hence $d_{i} d_{j}=0$ for $2 \leq i, j \leq n$ and $d_{1} d_{k}=0$ for $2 \leq k \leq n$. Therefore

$$
\mathcal{O}_{k}=-d_{k} d_{1}=\left\{\begin{array}{cc}
-x_{6 k-5} \frac{\partial}{\partial x_{6 k}}, & 2 \leq k<n, \\
0, & n=k .
\end{array}\right.
$$

On the other hand, $d_{k} d=0$ since $d(S) \subset S^{\perp}$; hence

$$
\delta\left(d_{k}\right)=d d_{k}
$$

It is now a simple matter to check that

$$
\delta\left(-d_{k+1}\right)=-x_{6 k-5} \frac{\partial}{\partial x_{6 k}}=\mathcal{O}_{k}
$$

for $2 \leq k<n$. Now for all $i>n$, set $d_{i}=0$ so that $\mathcal{O}_{i}=-d_{i} d_{1}=0$ and obtain a polynomial deformation $V_{t}=\left(V[[t]], d+t d_{1}+t^{2} d_{2}+\cdots+t^{n} d_{n}\right)$ of $(V, d)$. To establish non-triviality, assume that $\phi_{t}: V_{t} \sim V_{0}[[t]]$. Then there exists a cochain $\phi_{1} \in C^{0}(V)$ such that $\delta\left(\phi_{1}\right)=-d_{1}$, i.e.,

$$
d \phi_{1}-\phi_{1} d=-x_{1} \frac{\partial}{\partial x_{4}}-x_{4} \frac{\partial}{\partial x_{6}}+\text { (other terms). }
$$

Now $d\left(x_{6}\right)=0$ so $\delta\left(\phi_{1}\right)\left(x_{6}\right)=d \phi_{1}\left(x_{6}\right)=-x_{4}$. But this is a contradiction since $\phi_{1}\left(x_{6}\right) \in V_{3}=\left\langle x_{5}, x_{6}\right\rangle$ and $d\left(V_{3}\right)=0$. Finally, if we redefine $d_{1}=x_{4} \frac{\partial}{\partial x_{6}}$, then $d_{t}=d+t d_{1}$ is a non-trivial linear deformation, as the reader can easily check. At the other extreme, redefine $d_{1}=x_{1} \frac{\partial}{\partial x_{4}}+\sum_{i=1}^{\infty} x_{6 i-2} \frac{\partial}{\partial x_{6 i}}$ and obtain a non-trivial deformation with non-zero terms of all orders. We have proved:

Theorem 3.1. For each $n \geq 1$, there exists a d.g.m $(V, d)$ and a non-trivial polynomial deformation of order $n$. Furthermore, a sequence $\left\{d_{i}\right\}_{i \geq 1}$ can be chosen such that $V_{t}=\left(V[[t]], d_{t}=d+t d_{1}+t^{2} d_{2}+\cdots\right)$ is a non-trivial deformation with $d_{i} \neq 0$ for all $i$.

We conclude by constructing an approximation to a differential on $V[[t]]$ that is obstructed at order $n$. First assume that $2 \leq k<n$ and define $d_{1}$ and $d_{k}$ as in the example above. Extend $d+t d_{1}$ to an approximation of order $n-1$ in the same manner as before and obtain $\mathcal{O}_{n-1}=-d_{n-1} d_{1}=-x_{6 n-11} \frac{\partial}{\partial x_{6 n-6}}$. Define

$$
d_{n}=-x_{6 n-9} \frac{\partial}{\partial x_{6 n-6}}+x_{6 n-6} \frac{\partial}{\partial x_{6 n-4}}
$$


and observe that $\delta\left(d_{n}\right)=\mathcal{O}_{n-1}$ so that the approximation of order $n-1$ extends to order $n$. This time,

so that

$$
\mathcal{O}_{n}=-d_{1} d_{n}=-x_{6 n-8} \frac{\partial}{\partial x_{6 n-4}}
$$

$$
\mathcal{O}_{n}(V) \subset V_{3 n-4}
$$

Since $d(V) \subset \sum_{i=1}^{\infty} V_{3 p-2}$, we have

$$
\delta(f)(V)=(d f+f d)(V) \subset \sum_{p=1}^{\infty} V_{3 p-2} \oplus V_{3 p},
$$

for all $f \in C^{1}(V)$. Consequently $\mathcal{O}_{n}$ fails to cobound and the approximation is obstructed at order $n$. Finally, when $n=1$, define $d_{1}=x_{4} \frac{\partial}{\partial x_{6}}+x_{6} \frac{\partial}{\partial x_{8}}$ and observe that $\delta\left(d_{1}\right)=0$ while $\mathcal{O}_{1}=-d_{1}^{2}=-x_{4} \frac{\partial}{\partial x_{8}}$ fails to cobound since $\mathcal{O}_{1}(V) \subset V_{2}$. This proves our main result:

Theorem 3.2. For each $n \geq 1$, there exists a differential graded $\mathbf{k}$-module $(V, d)$ and an obstructed approximation to a differential $d+t d_{1}+t^{2} d_{2}+\cdots t^{n} d_{n}$ on $V[[t]]$.

\section{ACKnowledgement}

We wish to thank Murray Gerstenhaber for his helpful comments and suggestions.

\section{REFERENCES}

1. D. Burghelea and M. V. Poirrier, Cyclic Homology of Commutative Algebras I, in (Y. Felix, ed.) Proc. Louvain-la-Neuve 1986, Springer-Verlag, Berlin (1988), LNM Vol. 1318, 51-72. MR 89k:18027

2. Y. Felix, Dénombrement des Types de K-Homotopie. Théorie de la Déformation, Mémoire de la Société Mathématique de France (N. S.), n ${ }^{o} 3$ (1980). MR 82i:55011

3. M. Gerstenhaber and S. D. Schack, Algebras, Bialgebras, Quantum groups, and Algebraic Deformations, Contemporary Math. 134 AMS, Providence (1992), 51-92. MR 94b:16045

4. Applied Alg. 43 (1986), 199-222. MR 88a:16045

5. M. Gerstenhaber and C. Wilkerson, On the Deformation of Rings and Algebras, V: Deformation of Differential Graded Algebras, Contemporary Math. 227 AMS, Providence (1998), 89-101. MR 2000a: 16056

6. S. Halperin and J. Stasheff, Obstructions to Homotopy Equivalences, Adv. in Math. 32 (1979), 233-279. MR 80j:55016

7. M. Markl, A Cohomology Theory for $A(m)$-algebras and Applications, J. Pure and Applied Alg. 83 (1992), 141-175. MR 94a:18012

8. R. Umble, The Deformation Complex for Differential Graded Hopf Algebras, J. Pure and Applied Alg., 106 (1996), 199-222. MR 97f:16021

Department of Health Evaluation Sciences, Penn State College of Medicine, MC

H173, P.O. Box 850, 500 University Dr., Hershey, Pennsylvania 17033

E-mail address: tja3@psu.edu

Department of Mathematics, Millersville University of Pennsylvania, Millersville, Pennsylvania 17551

E-mail address: Ron.Umble@millersville.edu 